\title{
Autoconceito, depressão e ansiedade de pessoas em situação de rua
}

\author{
Self-concept, depression and anxiety of street people
}

\author{
Maria Girlane Sousa Albuquerque Brandão' • Maria Aline Moreira Ximenes ${ }^{2}$ \\ Caroline Ponte Aragão ${ }^{3}$ Joselany Áfio Caetano ${ }^{4}$ \\ Thiago Moura de Araújo ${ }^{5}$ Lívia Moreira Barros ${ }^{6}$
}

\begin{abstract}
RESUMO
O objetivo do estudo é avaliar o autoconceito e nível de depressão e ansiedade de pessoas em situação de rua. Trata-se de estudo exploratório descritivo realizado com pessoas em situação de rua de Centro para População em situação de rua no interior do Ceará, Brasil. Das pessoas atendidas pelo Centro, 33 apresentaram disponibilidade. Foram utilizados quatro instrumentos para coleta de dados (Questionário sociodemográfico, Inventário Clínico de Autoconceito, Inventário de depressão de Beck e Inventário de Ansiedade de Beck).As maiores médias nos domínios de autoconceito foram relacionados à autoeficácia e aceitação social. Com relação às facetas de autoconceito verificou-se como fatores positivos ser competente $(3,97 \pm 0,68)$, enfrentador $(3,85 \pm 0,83)$ e verdadeiro $(3,85 \pm 0,83)$. Como facetas negativas: bem-estar diminuído $(2,42 \pm 0,96)$, pouco falador $(2,70 \pm 1,18)$ e embirrento $(3,18 \pm 0,80)$. Quando ponderados os pontos obtidos no Inventário de depressão, verificou-se grau severo de depressão na maioria dos participantes $(39,4 \%)$. Na avaliação dos pontos obtidos no Inventário de ansiedade, verificou-se grau moderado de ansiedade em 39,4\% dos participantes. É fundamental identificar o autoconceito e os níveis de depressão e ansiedade dessa clientela, além de refletir sobre suas problemáticas e necessidade de apoio integral, diferenciado e livre de preconceitos.
\end{abstract}

Palavras-Chave: Depressão;Ansiedade; Saúde mental; Pessoas em situação de rua.

\begin{abstract}
The aim of the study is to evaluate the self-concept and level of depression and anxiety of people on the street. This is a descriptive exploratory study carried out with people living in the street from the Center for Population in a street situation in the interior of Ceará, Brazil. Of the people served by the Center, 33 were available. Four instruments were used for data collection (Sociodemographic Questionnaire, Clinical Inventory of Self-concept, Beck Depression Inventory and Beck Anxiety Inventory). The highest means in the self-concept domains were related to self-efficacy and social acceptance. Regarding the facets of self-concept, positive factors were found to be competent $(3,97 \pm 0,68)$, opposing $(3,85 \pm 0,83)$ and true $(3,85 \pm 0,83)$. As negative facets: decreased well-being $(2,42 \pm 0,96)$, not very talkative $(2,70 \pm I, I 8)$ and cheeky $(3,18 \pm 0,80)$. When weighted the points obtained in the Inventory of depression, there was a severe degree of depression in the majority of participants $(39,4 \%)$. In the evaluation of the points obtained in the Anxiety Inventory, there was a moderate degree of anxiety in $39,4 \%$ of the participants. It is essential to identify the self-concept and the levels of depression and anxiety of this clientele, in addition to reflecting on their problems and the need for integral, differentiated and free of prejudice.
\end{abstract}

Keywords: Depression, anxiety, mental health, street people. 


\section{INTRODUÇÃO}

Pessoas em situação rua (PsR) utilizam-se de estabelecimentos públicos como local de moradia. O quantitativo cada vez maior desse grupo populacional promove preocupações sociais contínuas em todo o mundo. Os números estimam que haja mais de 600 mil pessoas em sofrimento por falta de moradia nos EUA e mais de 400 mil pessoas na União Europeia. No Brasil, dados do Instituto de Pesquisa Econômica Aplicada (IPEA) indicam que 101.854 pessoas estavam em situação de rua em 2015(I).

Embora este seja um grupo heterogêneo, agregam em comum a fragilidade dos vínculos sociais, violências, preconceitos, descriminações, falta de privacidade, carências de educação e de infraestrutura para os cuidados corporais. Tais situações de vulnerabilidade colaboram para $\circ$ aparecimento e agravamento de muitos problemas, principalmente, os transtornos mentais e distúrbios no autoconceito que, por sua vez, podem ser um dos fatores que contribuem para que uma pessoa viva em situação de rua (2). Os estereótipos relativos às PsR são bastante negativos, o que pode provocar a diminuição do autoconceito e autoestima dessas pessoas, além de favorecer o surgimento da depressão e ansiedade.

Diante da realidade a que estão expostos, PsR apresentam fatores de risco que potencializam o desenvolvimento de transtornos mentais. A depressão e ansiedade são comuns entre pessoas em situação de rua e podem interferir no seguimento de tratamentos, em sua reinserção social, impedir a conquista de uma moradia estável, obtenção de emprego e até mesmo a procura a serviços de saúde ${ }^{(3)}$.

O fato de PsR estarem em contexto de maior vulnerabilidade e pressão social, estas encontram-se com maior probabilidade de desenvolver algum transtorno mental. Contudo, é válido salientar que isso não as impõe um destino imutável ou uma realidade estática. É imperativo que a sociedade, especial os profissionais de saúde, busquem compreender essa clientela, entender as suas peculiaridades e ofertar equidade e resolubilidade a seus problemas de saúde ${ }^{(4)}$.

Nessa perspectiva, cabe aos profissionais de saúde conhecer o contexto dessa população, para adaptar suas intervenções, conforme as particularidades existentes e às deficiências identificadas. Portanto, torna-se relevante a realização de estudos que visem identificar a presença de transtornos como ansiedade e depressão nessa população.

Ao reconhecer transtornos mentais em pessoas em situação de rua, como depressão e ansiedade, os profissionais podem exercer atendimento individual com planejamento de condutas e analisar o indivíduo em sua integralidade, além de contribuir para o cuidado de saúde mental efetivo diante das necessidades de cada ser humano.

Assim, essa pesquisa teve como objetivo avaliar o autoconceito e nível de depressão e ansiedade de pessoas em situação de rua.

\section{MÉTODO}

Estudo do tipo exploratório descritivo realizado com população adulta em situação de rua de cidade do interior do Ceará, Brasil. Os participantes foram selecionados por meio de amostra aleatória, dentre a população em situação de rua que frequentava o Centro de Referência Especializado para a População em Situação de Rua (Centro POP), no período de maio a dezembro de 2018 .

O cenário da pesquisa é um serviço vinculado à Secretaria dos Direitos Humanos, Habitação e Assistência Social de cidade da Região Norte do Estado do Ceará, que tem por objetivo potencializar a capacidade de proteção da família e promover a reparação da situação vivida. Trata-se de serviço público diurno de acesso aberto, com atividades diferenciadas de acordo com o turno, como atividades culturais, rodas de conversa e oficinas socioeducativas com apoio do Centro de Atenção Psicossocial (CAPS).

A população-alvo do estudo foram 60 pessoas em situação de rua atendidas pelo referido Centro POP, que acolhe PsR desde 20I4. Das 60 pessoas em situação de rua atendidas pelo Centro POP, 33 apresentaram disponibilidade para participar e, assim, compuseram a amostra da pesquisa. Foram delineados como critérios de inclusão: pessoas em situação de rua que frequentavam o Centro POP, com idade igual ou superior a 18 anos. Os critérios elegidos para exclusão foram: ter comprometimento cognitivo ou estar sob efeito de agentes psicotrópicos, o que poderia acarretar viés de seleção ao estudo.

Foram utilizados quatro instrumentos para coleta de dados. Primeiro ocorreu à aplicação de questionário sociodemográfico com 10 questões para coletar dados inerentes ao perfil dos participantes. No segundo momento, aplicou-se o Inventário Clínico de Autoconceito, em terceiro, o Inventário de depressão de Beck, e por fim, o Inventário de Ansiedade de Beck. O Inventário Clínico do Autoconceito (ICAQ) foi criado porVazSerra em 1985 e refere-se a aspectos sociais e emocionais considerados importantes no ajustamento pessoal. Trata-se de escala tipo Likert, com 20 questões de autoavaliação que devem ser respondidas segundo cinco modalidades: I - não concordo, 2- concordo pouco, 3 - concordo moderadamente, 4- concordo muito e 5 - concordo muitíssimo(5).

Cada modalidade é codificada de I a 5 , com exceção de três itens em que, por estarem redigidas de forma negativa, as pontuações devem ser revertidas (itens 3, 12 e 18). Quanto maior a pontuação, melhor e mais favorável é o autoconceito do indivíduo. $O$ instrumento também permite avaliar quatro fatores: (I) Aceitação/Rejeição social (itens I, 4, 9, 16 e 17); (2) Autoeficácia (itens 3, 5, 8, II, 18 e 20); (3) Maturidade Psicológica (itens 2, 6,7, I3), e (4) Impulsividade-atividade (itens 10, 15 e 19). Existem ainda dois fatores (itens 12 e 14) com carácter misto, os quais os autores não atribuíram nome $e^{(5)}$.

O inventário de depressão de Beck (BDI) trata-se de escala de autoavaliação de depressão, com 21 dimensões de sintomas, que abarcam os componentes cognitivos, afetivos, comportamentais e somáticos da depressão, com valores que variam de 0 a 3 pontos em escala Likert, na qual $\circ$ participante identifica a afirmativa que melhor descreve seu estado emocional vivenciado nas duas últimas semanas. As classificações dos sintomas podem ser normais (0-II pontos), leves ( $12-19$ pontos), moderados (20-35 pontos) e severos (36-63 pontos) $)^{(6)}$.

O inventário de ansiedade de Beck (BAI) é uma escala de autorrelato, com avaliação da intensidade de sintomas de ansiedade, composta por 21 itens. Compõe-se de escala subjetiva do tipo Likert com quatro pontos, com variação de 0 a 3 , em que o participante autoavalia o grau do sintoma comum à ansiedade. $O$ escore total é obtido por meio do somatório dos escores dos itens individuais, que permitem a classificação dos níveis de intensidade da ansiedade normal (0 - 10 pontos), leve (1I - 19 pontos), moderado (20 - 30 pontos) e severo (3I - 63 pontos) ${ }^{(6)}$. 
Os instrumentos foram aplicados em sala reservada do Centro POP no horário de funcionamento do serviço (08:00 às 17:00), com objetivo de assegurar ambiente privativo e seguro. Antes de cada entrevista houve a explicação do objetivo da pesquisa e todos os participantes assinaram o Termo de Consentimento Livre e Esclarecido (TCLE).

Os dados da pesquisa foram organizados e tabulados no programa Excel 2016 e analisados no programa estatístico Statistical Package for the Social Sciences (SPSS) versão 25. A análise estatística descritiva das variáveis categóricas foi apresentada em frequências absolutas e relativas e, para as variáveis contínuas, utilizou-se média e desvio-padrão.

O estudo foi aprovado pelo Comitê de Ética em Pesquisa (CEP) da Universidade Estadual Vale do Acaraú $\left(n^{\circ} 2.083 .621 / 2017\right)$ e atendeu todos os preceitos éticos da Resolução n 466/I 2 do Conselho Nacional de Saúde.

\section{RESULTADOS}

Todos os participantes do estudo eram do sexo masculino $(100 \%)$ com idade média de $36,33( \pm 8,66)$ e a maioria $(63,6 \%)$ possuía filhos. O grau de escolaridade predominante foi o ensino fundamental incompleto $(42,4 \%)$ seguido do médio incompleto $(21,2 \%)$. Em relação ao estado civil, foram identificados solteiros $(72,7 \%)$, casados $(9,1 \%)$, divorciados ( $15,2 \%)$, e viúvos (3\%). Em relação à religião, católicos $(42,4 \%)$, evangélicos (15,2\%), ateus (39,4\%) e adventistas (3\%). No tocante a renda, houve superioridade da ausência de renda mensal $(60,6 \%)$ e $33,3 \%$ sustentam-se de programas de assistencialismo social, em que recebem ao início de cada mês o valor monetário de 80,00 . Quanto à procedência dos participantes, houve predomínio da cidade de Sobral $(45,5 \%)$, seguidos pela macrorregião de Sobral $(24,2 \%)$, outros estados $(21,2 \%)$ e Fortaleza $(9,1 \%)$.

$\mathrm{Na}$ tabela I, apresentam-se as médias dos domínios relacionados ao autoconceito.

Tabela I. Distribuição dos domínios de autoconceito de Pessoas em Situação de Rua do Centro POP pelo ICAQ. Sobral (CE). Brasil. 2018.

\begin{tabular}{|l|l|}
\hline \multicolumn{1}{|c|}{ DOMÍNIOS } & \multicolumn{1}{c|}{ Média (Desvio Padrão) } \\
\hline Aceitação social & $I 5,64( \pm 2,88)$ \\
\hline Autoeficácia & $21,73( \pm 3,54)$ \\
\hline Maturidade psicológica & $I 4,3( \pm I, 72)$ \\
\hline Impulsividade & $7,15( \pm I, 27)$ \\
\hline Misto I & $3,18( \pm 0,80)$ \\
\hline Misto 2 & $3,97( \pm 0,68)$ \\
\hline \multicolumn{2}{|c|}{ Fonte: Dados da Pesquisa, 2018. }
\end{tabular}

Foi possível observar que as pessoas em situação de rua apresentaram maiores médias nos domínios relacionados à autoeficácia e aceitação social (Tabela I).

Tabela 2. Pontuações das variáveis de autoconceito de Pessoas em Situação de Rua do Centro POP pelo ICAQ. Sobral (CE). Brasil. 2018.

\begin{tabular}{|ll|l|}
\hline \multicolumn{2}{|c|}{ Variáveis ICAQ } & \multicolumn{1}{c|}{ Média (Desvio Padrão) } \\
\hline I. & Simpático & $3,61(0,82)$ \\
\hline 2. & Franco & $3,52(0,90)$ \\
\hline 3. & Não desistente & $3,64(1,27)$ \\
\hline 4. & Falador & $2,70(1,18)$ \\
\hline
\end{tabular}

\begin{tabular}{|l|l|}
\hline 5. $\quad$ Rápido & $3,73(0,83)$ \\
\hline 6. $\quad$ Tolerante & $3,24(1,06)$ \\
\hline 7. $\quad$ Responsável & $3,82(0,72)$ \\
\hline 8. $\quad$ Enfrentador & $3,85(0,83)$ \\
\hline 9. Aceitável & $3,24(1,1 \mathrm{I})$ \\
\hline I0. Pragmático & $3,42(1,03)$ \\
\hline I1. Persistente & $3,70(0,84)$ \\
\hline I2. Não-embirrento & $3,18(0,80)$ \\
\hline I3. Verdadeiro & $3,85(0,83)$ \\
\hline I4. Competente & $3,97(0,68)$ \\
\hline I5. Fazer/apetecer & $3,73(0,62)$ \\
\hline I6. Bem-estar & $2,42(0,96)$ \\
\hline I7. Agradável & $3,67(0,77)$ \\
\hline I8. Não-dependente & $3,42(0,83)$ \\
\hline I9. Bem sucedido & $3,70(0,84)$ \\
\hline 20. Enérgico & $3,39(0,99)$ \\
\hline
\end{tabular}

Fonte: Dados da Pesquisa, 2018.

Com relação às facetas na tabela 2 , verificou-se como fatores positivos ser competente $(3,97 \pm 0,68)$, enfrentador $(3,85 \pm 0,83)$ e verdadeiro $(3,85 \pm 0,83)$. Como facetas negativas: bem-estar diminuído $(2,42 \pm 0,96)$, pouco falador $(2,70 \pm I, I 8)$ e embirrento $(3,18 \pm 0,80)$.

Quando ponderados os pontos obtidos no BDI, verificouse que apenas um participante foi classificado com sintomas normais, enquanto nove $(27,3 \%)$ pessoas em situação de rua foram classificadas com sintomas depressivos leves. Foi constatado grau moderado em 30,3\%, seguindo-se de grau severo acometendo a maioria dos participantes (39,4\%). A ocorrência e a gravidade dos sintomas determinados por meio da aplicação do BDI podem ser visualizadas na tabela 3 .

Tabela 3. Distribuição da classificação dos sintomas de depressão de Pessoas em Situação de Rua do Centro POP. Sobral (CE). Brasil. 2018.

\begin{tabular}{|l|c|c|}
\hline Classificação dos sintomas & $\mathrm{N}$ & $\%$ \\
\hline Depressão (BDI) & 01 & 3,0 \\
\hline Normais & 09 & 27,3 \\
\hline Leves & 10 & 30,3 \\
\hline Moderados & 13 & 39,4 \\
\hline Severos & \multicolumn{2}{|c|}{ Fonte: Dados da Pesquisa, 2018. }
\end{tabular}

$\mathrm{Na}$ avaliação dos pontos obtidos no BAl, verificou-se que quatro $(12,1 \%)$ participantes foram classificados com sintomas normais, oito $(24,2 \%)$ com sintomas de ansiedade leves. Verificou-se grau moderado de ansiedade em 13 participantes $(39,4 \%)$, seguindo-se de grau de ansiedade severo em oito participantes $(24,2 \%)$, conforme a tabela 4 .

Tabela 4. Distribuição da classificação dos sintomas de ansiedade de Pessoas em Situação de Rua do Centro POP. Sobral (CE). Brasil. 2018.

\begin{tabular}{|l|c|c|}
\hline Classificação dos sintomas & $\mathrm{N}$ & $\%$ \\
\hline Ansiedade (BAI) & 04 & $12, \mathrm{I}$ \\
\hline Normais & 08 & 24,2 \\
\hline Leves &
\end{tabular}




\begin{tabular}{|l|c|c|}
\hline Moderados & 13 & 39,4 \\
\hline Severos & 08 & 24,2 \\
\hline \multicolumn{2}{|c|}{ Fonte: Dados da Pesquisa, 2018. }
\end{tabular}

\section{DISCUSSÃO}

A maioria da amostra foi composta por homens solteiros $(69,7 \%)$, adultos jovens com média de idade de $36,33( \pm 8,66)$ e ensino fundamental incompleto $(42,4 \%)$. Tais achados corroboram com outras investigações nacionais ${ }^{(4-7)}$ e internacionais ${ }^{(8-9)}$. Isso demonstra que esse grupo populacional apresenta características semelhantes em diversos contextos sociais e localizações geográficas, tendo em comum a ausência de moradia convencional regular e a rua como o espaço de habitação e sustento, onde impera a necessidade de pensar novos recursos que favoreçam as condições de vida e saúde dessa clientela.

Viver nas ruas configura-se como fenômeno que afeta diversos países pelo mundo.Tal problema está associado a uma série de determinações, como precarização ou rompimento total das relações de trabalho formal e a ruptura dos vínculos familiares e comunitários. Estes determinantes originam prejuízos ao suporte familiar e comunitário e da identidade social de trabalhador e a sobrevivência em condições subdignas $^{(10)}$.

Assim, a situação de rua está relacionada a condições de extrema miséria em que se têm negados direitos fundamentais. A maioria dos participantes do estudo $(60,6 \%)$ não possui nenhuma renda que possibilite o custeio de necessidades básicas como alimentação e higiene pessoal e 33,3\% vivem amparados por 80,00 mensais que recebem de programas de assistencialismo social. Esse dado corrobora com estudo censitário realizado em 7l cidades brasileiras, que revelou a existência de 31.922 adultos vivendo em situação de rua, com níveis de renda baixo em atividades precárias de geração de renda na rua, a maioria (52,6\%) recebia entre 20,00 e 80,00 semanais( ${ }^{(I)}$. A situação de baixa renda que aflige a maioria das PsR está comumente associada a baixa escolaridade desse público, poucos possuem o ensino fundamental completo, que impende, na maioria das vezes, a obtenção de emprego assalariado com registro formal, além de outras causas, como preconceito e estigma social.

Estudo realizado em Bangladesh, na Ásia, revelou que o estilo de vida de muitas PsR, sem oportunidade de trabalho e renda, déficits no estado de saúde, hábito alimentar e instalações de moradia precárias podem gerar sérios problemas físicos e mentais ${ }^{(12)}$. Esse resultado traduz as dificuldades e mazelas sofridas por muitas PsR, a maioria vive privada de atividades de lazer, com poucos recursos financeiros e falta de segurança (muitos carregam no corpo as marcas das violências sofridas), fatores preditivos de baixa qualidade de vida, com aumento do estresse e exaustão emocional, que se refletem em sua saúde mental. Neste estudo, a autoeficácia e aceitação social foram os domínios do autoconceito com maiores médias. Estudo realizado com II 4 residentes de centros de acomodação para PsR na Austrália, evidenciou que $31 \%$ dos entrevistados não se auto categorizavam com morador de rua e este fator esteve associado a menores sintomas negativos de humor e melhor eficácia em atividades cotidianas. Isso sugere que rejeitar uma autocategorização de pessoa em situação de rua pode ser uma estratégia protetora para amortecer o autoconceito e o bemestar psicológico de uma pessoa das implicações negativas de pertencer a um grupo estigmatizado(13).
Quanto aos principais fatores positivos relacionados ao autoconceito destaca-se ser competente $(3,97 \pm 0,68)$, enfrentador $(3,85 \pm 0,83)$ e verdadeiro $(3,85 \pm 0,83)$. Esta análise apoia a afirmação de que, apesar de suas vulnerabilidades e limitações, as PsR têm atributos positivos relacionados a auto percepção. No entanto, no que diz respeito aos aspectos negativos, foi evidenciado o bem-estar diminuído $(2,42 \pm 0,96)$, a fala diminuída $(2,70 \pm I, 18)$ e comportamento embirrento $(3,18 \pm 0,80)$. Dessa forma, devem ser realizadas intervenções focadas nas facetas negativas, como forma de melhorar o bemestar e as relações interpessoais. Da mesma forma, devem ser reforçadas as facetas positivas para aumentar ainda mais - capital psicológico e contribuir para o autoconceito desse público.

Apesar da situação de rua proporcionar imersão desses sujeitos a condições de diminuição e invisibilidade, a construção de uma percepção positiva de si mesmo e reconhecimento de peculiaridades positivas em suas personalidades pode facilitar a construção de um sentido a vida e motivação para superar as dificuldades. Todo ser humano, independentemente de circunstâncias socioeconômicas, psíquicas ou culturas, é capaz de construir significações existenciais positivas com base em seu autoconceito ${ }^{(14)}$.

No que diz respeito aos transtornos mentais, a maioria dos participantes apresentaram grau severo ou moderado de depressão. Pesquisa que buscou identificar a prevalência de depressão entre 245 homens adultos em situação de rua em Belo Horizonte revelou que 60 (24,5\%) apresentaram grau moderado de depressão e $12(4,9 \%)$ sintomas depressivos graves $^{(4)}$.

A depressão é um transtorno multifatorial que apresenta fatores de risco conhecidos, como afetividade negativa, eventos estressantes, rompimento de laços familiares e transtornos subjacentes $^{(15)}$. No caso de PsR esse se torna um problema sério por viverem em ambientes estressantes extremamente vulneráveis, em que é imperativo fortalecer a política de saúde mental, além de desenvolver novas estratégias que favoreçam a acessibilidade em todos os serviços de saúde e nos centros de referência para população em situação de rua.

$\mathrm{Na}$ avaliação do inventário de ansiedade, verificou-se predomínio de ansiedade moderada. Pesquisa realizada com 309 homens em situação de rua nos EUA, evidenciou que um em cada três homens atingem o limiar de depressão moderada a grave. Além do mais, os homens que estavam deprimidos eram mais propensos ao abuso de substâncias ilícitas e a manifestação de problemas de saúde físicos ${ }^{(3)}$. Estudo realizado em João Pessoa com 100 pessoas em situação de rua verificou que $30 \%$ estavam com depressão mínima, $44 \%$ leve e $26 \%$ moderada. $A$ ansiedade atingiu 37\% em nível mínimo, 38\% leve, $20 \%$ moderada, $5 \%$ grave $^{(16)}$.

O acompanhamento das pessoas em situação de rua com transtorno mental é permeada por desafios referentes ao próprio público e a ausência de preparo formativo de profissionais no âmbito da saúde mental, em que há necessidade urgente de melhorias nas ações e articulações intersetoriais, além de maximizar recursos humanos, estruturais e materiais ${ }^{(17)}$.

Estes avanços, principalmente no contexto da Atenção Primária à Saúde, bem como, o acesso a cuidados médicos apropriados, podem ajudar a melhorar os resultados sociais e minimizar os quadros de ansiedade nessa população vulnerável, os quais podem desencadear inúmeros transtornos psíquicos $^{(18)}$. Destaca-se ainda, a importância não somente do 
acesso a cuidados médicos, mas da qualidade destes cuidados. As pessoas em situação de rua são constantemente tratadas como seres ociosos, o que promove a perda de confiança, rompimento de vínculos e isolamento social(19).

Revisão de literatura que analisou 704 artigos sobre assistência a PsR delineou alguns métodos eficazes no tratamento de problemas com PsR, inclusive transtornos mentais, como depressão e ansiedade. Dentre estas estratégias utilizadas, destacou-se a orientação clínica, que melhorou - acesso ao sistema de saúde, modelos multidisciplinares baseados em equipe, incluindo médicos de atenção primária e enfermeiros clínicos de apoio social (apoio à habitação, serviços sociais localizados e disponibilidade de necessidades básicas no local) e envolvimento na saúde da comunidade(20).

Assim, o aumento da densidade das redes de apoio social pode minimizar o risco de sofrimento mental de pessoas em situação de rua(4).

\section{CONCLUSÃO}

A situação de rua acomete predominantemente homens solteiros com grau severo de depressão e grau moderado de sintomas ansiosos. É fundamental identificar o autoconceito e os níveis de depressão e ansiedade destes indivíduos, além de refletir sobre suas problemáticas e necessidade de apoio integral, diferenciado e livre de preconceitos. Logo, os resultados deste estudo podem subsidiar ações direcionadas a este público, posto que grande parte das pessoas que vivenciam a situação de rua tem seus cuidados de saúde mental negligenciados.

Destaca-se a limitação do público somente com pessoas em situação de rua frequentadoras do Centro POP, em que não é possível generalizar os resultados aos indivíduos que não buscam o serviço. Ressalta-se ainda, que existe uma escassez de estudos nacionais sobre transtornos mentais em pessoas em situação de rua, em que se torna necessário a realização de pesquisas como fundamento científico para a prática assistencial às pessoas em situação de rua.

Espera-se que esse estudo venha contribuir com a qualidade da assistência em saúde às pessoas em situação de rua, por meio da identificação e atenção aos fatores que condicionam o surgimento de transtornos mentais, como depressão e ansiedade, e atender essa clientela de forma integral e individual.

\section{REFERÊNCIAS}

1. Natalino MAC. Estimativa da população em situação de rua no Brasil. Texto para discussão. Instituto de Pesquisa Econômica Aplicada. 2246 (I): I-36, 2016.

2. Costa AML, Engstrom EM, Ziliotto GC, Teixeira MB, Andrade GCL, Goldblum AV, et al. Práticas promotoras de saúde do Consultório de Rua na cidade do Rio de Janeiro: desafios do acesso e dos direitos sociais da população em situação de rua. São Paulo, ed. Hucitec, 2018.

3. Coohey C, Easton SD. Estressores distais e depressão entre homens desabrigados. Rev Trabalho Soc da Saúde. 20I6; 4 I (2): III-II9. http://dx.doi.org/|0.I590/SI4I3294X2007000100010

4. Botti NCL, Castro CG, Silva MF, Silva AK, Oliveira LC, Castro ACO, et al. Prevalência de depressão entre homens adultos em situação de rua em Belo Horizonte. J Bras Psiquiatr. 2010; 59 (I): 10-16. http://dx.doi.org/10.1590/ S0047-20852010000100002
5. FrancoV,Bacelar-Nicolau H.Autoconceito dos professores: principais factores usando modelos de Análise de Dados Multivariada. Educ rev. 2008; (I0) 32: I6I-I79. http:// dx.doi.org/ I0.1590/S0 I04-406020080002000 I2

6. Carvalho-Freitas MN, Silva VA, Tette RPG, Veloso HS, Rocha PC. Retorno às atividades laborais entre amputados: Qualidade de vida no trabalho, depressão e ansiedade. Rev Psicologia: Organizações e Trabalho. 2018 ; 18 (4), 468-475. http://dx.doi.org/10.17652/rpot/2018.4.144I5

7. Lopes PR,Winkelmann MCC, Heidemann ITSB, Fernandes GCM, Dalmolin IS. Percepção das pessoas em situação de rua acerca da promoção da saúde. Rev Enferm Atual. 2019; 83(2I):54-60.

8. Janssens JP, Wuillemin T, Adler D, Jackson Y. Screening for tuberculosis in an urban shelter for homeless in Switzerland: a prospective study. BMC Infect Dis. 2017; 17 (I):347-356. http://dx.doi.org/ I0.1 I 86/s I 2879-0 I7-2449-y

9. Tinland A, Boyer L, Loubière S, Greacen T, Girard $\mathrm{V}$, Boucekine $M$, et al. Victimization and posttraumatic stress disorder in homeless women with mental illness are associated with depression, suicide, and quality of life. Neuropsychiatr Dis Treat. 20 I8; (4) 14: 2269-2279. https:// doi.org/10.2147/NDT.SI61377

10. Baggett TP, Hwang SW, O'Connell J, Porneala BC, Stringfellow EJ, Orav EJ, et al. Mortality among homeless adults in Boston: shifts in causes of death over a 15-year period. JAMA Intern Med. 2013; 173 (3): I89-195. https:// doi.org/I0.100I/jamainternmed.2013.1604

11. Brasil. Ministério do Desenvolvimento Social e Combate à Fome (BR). Rua:Aprendendo a contar: Pesquisa nacional sobre população em situação de rua. Brasília. (DF): Ministério do Desenvolvimento Social e Combate à Fome, Secretaria de Avaliação e Gestão da Informação; 2009.

12. Farid H, Mostari M. Lives and Livelihoods of Children Living In Street Situation in Dhaka City of Bangladesh. Bangladesh Res Pub J. 20 I5; I I ( ): 40-48.

13. Walter ZC, Jetten J, Parsell C, Dingle GA. The impact of self-categorizing as "Homeless" on well-being and service use.Analyses of Social Issues and Public Policy. 20 I 5; 15 (I) 333-356. http://dx.doi.org/ I 0. I I I I/asap. 12089

14. Castro LZ, Andrade MC, Loureiro R. A população em situação de rua e a busca pelo sentido da vida: uma questão de sobrevivência. Rev Pretextos. 2018; 3(6): 224-235.

15. Ferrari AJ, Charlson FJ, Norman RE, Patten SB, Freedman G, Murray CJ, et al. Burden of depressive disorders by country, sex, age, and year: findings from the global burden of disease study 2010. PLoS medicine. 2013; 10 (II):el00I547. http://dx.doi.org/I0.137I/journal. pmed. 1001547

16. Patrício ACFA, Leite MAP, Silva RF, Santos TD, Silva RAR. Mental health, HIV and rapid tests of homeless people: a cross-sectional study. Online Brazilian J of Nursing. 2019; 16 (I): 577-79. https://doi.org/ I0.I7665/I676-4285.20I76I48

17. Wijk LBV, Mângia EF. O cuidado a Pessoas em Situação de Rua pela Rede de Atenção Psicossocial da Sé. Rev Saúde em Debate. 2017; 4 I (I I5): I I30- I |42. https://doi. org/10.1590/0103-110420171I5II

18. Roze M, Vandentorren S, Waerden J, Melchior M. Factors associated with depression among homeless mothers. Results of the ENFAMS survey. J Affect Disord. 2018; I5(229):https://doi.org/3 |4-321.10.1016/j.jad.2017.12.053 19. Rae BE, Rees S. The perceptions of homeless people 
regarding their healthcare needs and experiences of receiving health care. J Adv Nurs. 2015; 7 I(9):2096-107. https://doi.org/I0.I I I I/jan.12675

20. Jego M, Abcaya J, Ștefan DE, Calvet-Montredon C, Gentile S. Improving Health Care Management in Primary Care for Homeless People: A Literature Review. Int Environ Res. 2018, 15(2): 309-320. https://doi.org/l0.3390/

Recebido: 2019-06-14 ijerph I 5020309 